\title{
Orthography, phonology, and meaning: Word features that give rise to feelings of familiarity in recognition
}

\author{
ANNE M. CLEARY \\ Iowa State University, Ames, Iowa
}

\begin{abstract}
In the present study, it is shown that participants can recognize test cues as resembling studied words even when these cues cannot be used to recall the words that they resemble. After studying a list of words, participants were given a cued recall test for which half of the cues resembled studied words on one particular feature dimension and half resembled nonstudied words on that dimension. In addition to trying to use each cue to recall a study list item, participants rated the degree to which the cue resembled a studied word. For those cues whose targets could not be identified, the mean rating was higher when the cues corresponded to studied items than when they corresponded to nonstudied items. Various types of features can give rise to this phenomenon, which was found when orthographic, phonemic, and semantic cued recall tasks were used. In all of these cases of recognition without recall, analysis of receiver operating characteristics revealed a pattern consistent with that of an equalvariance signal detection process.
\end{abstract}

Over the years, researchers have devised many ways of examining how it is that people can recognize having experienced an event previously even when they cannot bring the specific prior episode to mind. Doing so requires teasing apart those instances in which recognition is based on recollection from those in which it is based on a more general sense of familiarity. Many theories posit a role of isolable features in the process of familiarity, yet very little is known about the specific types of features that can give rise to feelings of familiarity, especially when it comes to the recognition of single words from a study list. In the present study, a method of examining recognition is presented that may prove useful in addressing questions about the role of particular word features in familiarity.

Human memory theorists have long assumed that memory for a stimulus is essentially memory for a collection of the independent features present in that stimulus. This especially holds true for theories of how familiarity operates in recognition. Take, for example, the global matching approach to familiarity (see Clark \& Gronlund, 1996, for a review). According to this approach, familiarity is described as a signal that emerges from a matching process in which the separable features present in a given test item are matched with the features that have been

I thank Veronica J. Dark, Robert L. Greene, and Gary L. Wells for helpful comments on earlier versions of this article. I also thank Josette Barkley, Glenn Casner, Jodie Jutting, Erin Pierson, Kevin R. Seiler, and Mabel Skaug for assistance with data collection. Correspondence concerning this article should be addressed to A. M. Cleary, Department of Psychology, Iowa State University, W. 112 Lagomarcino, Ames, IA 50011 (e-mail: acleary@iastate.edu). stored in memory; the greater the degree of match, the stronger the familiarity signal will be. Because there will be a greater overall match for those test items that were studied than for those items that were not studied, the studied items will tend to evoke a stronger familiarity signal than those that were not studied, and thus provide a basis for discriminating between the two types of test items.

Theories that emphasize the use of separable item features in familiarity predict similarity effects in recognition. For example, because the match between the features present in each test item and the features present in memory is important to the global matching approach, it predicts that participants should tend to give false alarms to test items that are similar to studied items. In fact, many of the existing approaches to isolating familiarity from recollection capitalize on this idea (see Yonelinas, 2002, for a review).

One method of dissociating familiarity from recollection that involves manipulating similarity is the plurality task used by Hintzman and Curran (1994). In it, participants study a list of words that are presented in either their singular or plural forms (e.g., TABLE, LAKES). If a later recognition test requires that participants discriminate between studied (e.g., TABLE) and nonstudied (e.g., FROGS) items, they may rely either on the recollection of specific study items or on the level of familiarity evoked by each test item. If the recognition test requires participants to discriminate between words that have changed plurality from study to test (e.g., TABLES) and those that have remained the same (e.g., LAKES), participants should need to rely on recollection. Without recollection, participants should make a false alarm in response to a word 
that has changed plurality from study to test as a result of the sense of familiarity evoked by such a high degree of similarity.

Another method of dissociating familiarity from recollection has involved the use of compound words (e.g., Jones \& Jacoby, 2001). Here, participants study a list of compound words (e.g., BUCKWHEAT, JAILBIRD, BLACKMAIL). In addition to being presented with studied and nonstudied words at test, participants are presented with compound words that were not studied but contain root words that appeared in compound words that were studied. Some of these test words are recombined versions of studied words (e.g., BLACKBIRD), while some contain only one studied component (e.g., BUCKSHOT). The logic is similar to that used with the plurality task; the familiarity evoked by these latter two types of test items can lead to false alarms if recollection of the specific studied words does not occur to prevent it.

Although results obtained using these two tasks have been broadly consistent with featural approaches to the study of familiarity, they do not indicate how a wholeword unit might be separated into a set of features in memory for use in a later familiarity process. The plurality task involves using test words that are similar to studied words along many dimensions (e.g., orthography, phonology, and meaning); the only feature changing from study to test is the singular or plural status of the word. A similar limitation exists with the compoundword task in which the components used at test could be considered whole-word units themselves.

Are there particular features in a word that, if isolated from the whole-word unit, could give rise to a sense of familiarity? The present study examines whether such isolated features of words as their orthography, phonology, and meaning can evoke feelings of familiarity. Therefore, familiarity-based recognition must be separated from that which is recollection based. In the present study, a method of isolating familiarity is presented that explicitly allows for investigation of the types of features that may be used in this process. ${ }^{1}$ The approach taken here follows from that used to study recognition without identification (e.g., Peynircioğlu, 1990).

In each variant of the procedure reported here, participants study a list of words followed by a cued recall test for which half of the cues resemble studied words on one particular feature dimension, such as orthography (Variants 1 and 2), phonology (Variant 3), or meaning (Variant 4), and half resemble nonstudied words on that dimension. For each test cue presented, participants are first asked to try to use the cue to recall a similar item from the study list. Regardless of whether they are able to do so, they are then asked to rate the cue on a scale of 0 (definitely not similar to a studied word) to 10 (definitely similar to a studied word). The data of interest are the resemblance ratings given to cues whose targets could not be identified. If such separable features of words as their orthography, phonology, and meaning can, when presented in isolation, give rise to feelings of familiarity, then participants should be able to use this sense of familiarity to discriminate between the cues corresponding to studied words and those corresponding to nonstudied words in the absence of recall.

In search of support for the idea that such recognition without recall reflects familiarity-based recognition, the receiver operating characteristics (ROCs) for these data were examined. ROCs are often used to determine how well various aspects of recognition can be described by signal detection theory (e.g., Ratcliff, McKoon, \& Tindall, 1994). According to the Gaussian equal-variance signal detection model, the variances of the signal and noise distributions should be equal. Because the slope of the $z$-transformed ROC $(z$-ROC) reflects the ratio of the standard deviation of the noise distribution to that of the signal distribution, the Gaussian equal-variance signal detection model predicts a $z$-ROC slope of 1.0. However, recognition $z$-ROC slopes tend to be much lower than 1.0 ; most researchers have found slopes of or near .8 (e.g., Ratcliff et al., 1994), which would suggest that the variance of the signal distribution is greater than that of the noise distribution.

Yonelinas (1994) has argued that the usual slopes of less than 1.0 may result from the contribution of recollection to performance. That is, recollection would likely lead people to give high confidence ratings to a number of studied items (affecting the hit rate) without affecting how often they give high confidence ratings to nonstudied items (the false alarm rate), pulling the slope below 1.0. He argued that familiarity-based recognition alone is well described by simple signal detection theory and demonstrated that when the contribution of familiarity is isolated, the slope of the $z$-ROC approximates 1.0 (Yonelinas, 1994). Thus, if recognition without recall reflects the use of an equalvariance signal detection process, the $z$-ROCs for these data should be linear with a slope of approximately 1.0.

The data presented here were collected in four independent experiments. However, each of these experiments was a variant of the same general procedure, and the data from each were analyzed in the same manner. Therefore, for ease of exposition, they will be described as one large experiment with four independent conditions.

\section{METHOD}

\section{Participants}

In all variations of the procedure reported here, Iowa State University undergraduates participated in exchange for extra credit in an introductory psychology course. In the first variation, 19 students participated; in the second, 21 participated; in the third, 36 participated; and in the fourth, 29 participated.

\section{Materials}

In all variations of the experiment reported here, 60 words were randomly selected from a pool of 120 to be used as study list stimuli for each individual; in each variation, these items were selected from the same pool of possible study items across participants. One hundred twenty cues were used as test stimuli. Each of the cues was similar to a word from the pool of 120 potential study list words on one particular feature dimension, such as orthography (the first and 
second variations), phonology (the third variation), or meaning (the fourth variation). For the two orthographic variations, 120 words and their corresponding orthographically related cues were chosen from among those used by Blaxton (1989). So, for example, the word cheetohs might be used as an orthographically related cue for the study word cheetah. In the first of these two variations, both the study and test lists were presented visually, and in the second, the study list was heard, rather than seen. Because words that are orthographically similar also tend to be phonologically similar (e.g., cheetah and cheetohs), in the third variation, test cues that were phonologically but not orthographically similar to studied items were used. Half of the cues used at test rhymed with studied words and half rhymed with nonstudied words, and only words having rhymes dissimilar to them in orthography (e.g., raft, laughed; eighty, lady) were used. So, for example, laughed might serve as a test cue for the study word raft. In the fourth variation, the dimension of meaning was examined. Here, 120 words and their corresponding semantically related cues were chosen from among those used by Blaxton (1989). So, for example, the word jaguar might serve as a test cue for the study word cheetah.

In all four of the experimental variations reported here, the study and test stimuli were segmented into four study-test blocks. Each block consisted of a 15 -word study list followed by a 30 -item test list; 15 of the cues presented at test were similar to studied words on one particular feature dimension, while 15 were similar to nonstudied words on this dimension.

\section{Procedure}

All segments of a given variation of the procedure were conducted on a computer. Before the beginning of the experiment, the procedure was explained to the participants, each of whom completed four blocks, each consisting of a 15-word study list followed by a 30 -item test list. In the visual orthographic variation, the phonological variation, and the semantic variation, for which study words were presented visually, the words were presented for $2 \mathrm{sec}$ each at study, with an interstimulus interval of $1 \mathrm{sec}$. In the auditory orthographic variation, each study word was presented auditorily through a set of headphones and each was separated by a 1-sec interstimulus interval. In all of the variations, participants were instructed to remember each word presented during the study list. The nature of the memory test was also explained to them beforehand. They were told that, following the study list, they would be presented with a list of test words, and that half of these words would be similar to studied words and half would not. They were told that they would be asked to use each of these test words to try to recall a similar word from the study list. In addition, they were told that, regardless of whether they could recall a similar word, they would be asked to rate the likelihood that a similar word had appeared in the study list. More specifically, in both the visual and the auditory orthographic variations, they were told that half of the test words would look like studied words and half would not. In the phonological variation, they were told that half of the test words would rhyme with studied words and half would not. In the semantic variation, they were told that half of the test words would be similar in meaning to studied words and half would not.

During the test portion of a given block, each cue was presented singly at the top left corner of the computer monitor and remained on the screen as each of two questions was presented. First, participants were prompted to try to recall a similar word from the study list. For example, in the orthographic variations they were asked, "Do you recall a word from the study list that looked like this? If so, type the word." Participants could respond either by typing in a word and pressing the return key or by simply pressing the return key. After pressing return, they were prompted to rate the cue, indicating how likely they thought it was that a similar word had been presented on the study list. For example, in the visual orthographic variation, they were asked, "Do you think that a word looking like this appeared in the study list? Give a rating between 0 and $10(0=$ sure no; 10 = sure yes)." Participants could respond only by typing in an integer between 0 and 10 and then pressing the return key; if they typed a number larger than 10 or pressed the return key without typing a number, the question would be repeated. It was emphasized in the instructions that they should rate every cue presented at test, even when the cue could not be used to recall an item. For example, in the first variation participants were told, "Keep in mind that just because you could not recall a similar looking word from the study list doesn't mean that one did not appear on the list."

If a participant had successfully recalled an item in response to the first question (by typing the correct answer), then, after rating it, he or she was immediately presented with the next test cue. If, however, the participant had not typed in the correct word, then after giving a rating, he or she was given a second chance to recall the item. Here, if participants could not think of a similar word from the study list, they were requested to guess; for example, in the first variation, they were told to think of a similar-looking word and type it in. If a particular guess was correct, the rating was classified as corresponding to an identified item. This was done to ensure that any findings of recognition without recall would not be attributable to participants withholding responses during the recall portion of each test trial. Furthermore, the data were checked for spelling errors; the typed responses that accurately identified the target items but were spelled incorrectly were classified as correctly identified.

\section{RESULTS}

In all of the analyses presented here, a .05 significance criterion was used. The data for all experimental conditions are reported in Tables 1, 2, and 3; those of primary interest - the resemblance ratings given to the test cuesare listed in Table 2.

\section{Proportion of Cues Recalled}

Table 1 lists the cued recall data. The proportions listed under "Nonstudied" represent the instances in which participants correctly guessed the target word; that is, they typed the target word corresponding to the test cue (e.g., typed cheetah, in response to cheetohs) even though that word had not been presented at study. Table 1 can be summarized as showing that participants exhibited significant cued recall of the study list items in all variants of the experiment reported here. Participants identified significantly more targets of studied than of nonstudied cues in the visual orthographic variation $[t(18)=12.87]$, the auditory orthographic variation $[t(20)=12.46]$, the phonological variation $[t(28)=13.28]$, and the semantic variation $[t(35)=9.59]$. A $2 \times 4$ study status (studied vs. nonstudied) $\times$ segment (first vs. second vs. third vs. fourth) repeated measures ANOVA was done to examine recall performance across study-test segments. The only significant linear main effect of segment occurred

Table 1

Mean Proportion Items Correctly Identified in Each Condition

\begin{tabular}{llllll}
\hline & \multicolumn{2}{c}{ Studied } & & \multicolumn{2}{c}{ Nonstudied } \\
\cline { 2 - 3 } \multicolumn{1}{c}{ Condition } & $M$ & $S D$ & & $M$ & $S D$ \\
\hline Orthographic cue & .53 & .11 & & .16 & .08 \\
Modality switch/orthographic cue & .46 & .10 & & .13 & .06 \\
Rhyme cue & .18 & .09 & & .05 & .04 \\
Semantic cue & .33 & .13 & .04 & .03 \\
\hline
\end{tabular}


Table 2

Mean Resemblance Ratings Given to Test Cues in All Conditions

\begin{tabular}{|c|c|c|c|c|c|c|c|c|c|c|}
\hline \multirow[b]{3}{*}{ Condition } & \multicolumn{5}{|c|}{ Target Identified } & \multicolumn{5}{|c|}{ Target Unidentified } \\
\hline & \multicolumn{2}{|c|}{ Studied } & \multicolumn{2}{|c|}{ Nonstudied } & \multirow[b]{2}{*}{ Difference } & \multicolumn{2}{|c|}{ Studied } & \multicolumn{2}{|c|}{ Nonstudied } & \multirow[b]{2}{*}{ Difference } \\
\hline & $M$ & $S D$ & $M$ & $\overline{S D}$ & & $M$ & $S D$ & $M$ & $S D$ & \\
\hline Orthographic cue & 8.83 & 1.04 & 4.38 & 2.18 & 4.45 & 4.83 & 1.06 & 4.12 & 1.39 & .71 \\
\hline Modality switch/orthographic cue & 9.17 & 0.62 & 5.13 & 1.71 & 4.04 & 4.52 & 1.18 & 4.02 & 1.32 & .50 \\
\hline Rhyme cue & 8.14 & 1.56 & 1.88 & 3.91 & 6.26 & 4.46 & 1.47 & 4.15 & 1.29 & .31 \\
\hline Semantic cue & 9.20 & 0.80 & 4.41 & 2.47 & 4.79 & 4.16 & 1.29 & 3.92 & 1.23 & .24 \\
\hline
\end{tabular}

Note-Difference $=$ difference between the mean rating given to studied items and the mean rating given to nonstudied items.

in the auditory orthographic variation $[F(1,20)=6.68$, $p<.05]$; participants improved on the orthographic cued recall test as they moved through the segments of this variation, possibly because they began following a strategy during study of generating visual images of what the study words would look like in print. For the other variations, there were no such effects (orthographic variation $[F(1,18)=1.47$, n.s. $]$; phonological variation $[F(1,33)=$ 0.93 , n.s.]; semantic variation $[F(1,28)=1.00$, n.s. $]$ ).

\section{Resemblance Ratings}

The mean resemblance ratings are presented in Table 2. Those of interest are the resemblance ratings given to cues whose targets could not be identified. In all variations of the procedure reported here, when the test cues corresponding to studied words could not be used to recall the study words that they resembled, participants could still recognize that they resembled studied words. This was shown by the fact that participants gave higher recognition ratings to test cues corresponding to studied words than to test cues corresponding to nonstudied words, even when they could not successfully identify the targets corresponding to these cues. This effect was shown in the visual orthographic variation $[t(18)=3.25]$ and in the auditory orthographic variation, where the cues were orthographic in nature, but the presentation modality was changed from study to test $[t(20)=2.86]$. This effect was shown in the phonological variation, in which the cues were strictly phonological in nature (either rhyming with studied or nonstudied words but not looking like them) $[t(35)=2.50]$. Finally, the same effect was shown in the semantic variation (where the cues resembled either studied or nonstudied words in meaning) $[t(28)=2.71]$. Note that in all cases, each participant received a random subset of the 120 possible study words; thus, because a given test cue fell into both the "studied" and "nonstudied" categories across participants, the higher resemblance ratings given to cues of studied words can be seen as reflecting recognition of cue similarity to studied items, rather than a bias to give some items higher ratings than others. ${ }^{2}$

These resemblance ratings were used to compute the ROCs for the recognition without recall reported here; the $z$-ROCs were analyzed to determine how well this ability can be described by signal detection theory (see Figure 1). Linear regression was used to estimate the slopes and intercepts for the $z$-ROC curves. The goodness of fit for these linear regression equations $\left(R^{2}\right)$ was very near 1.0 in each variation. These $R^{2}$ values, as well as the slopes and intercepts, are presented in Table 3.
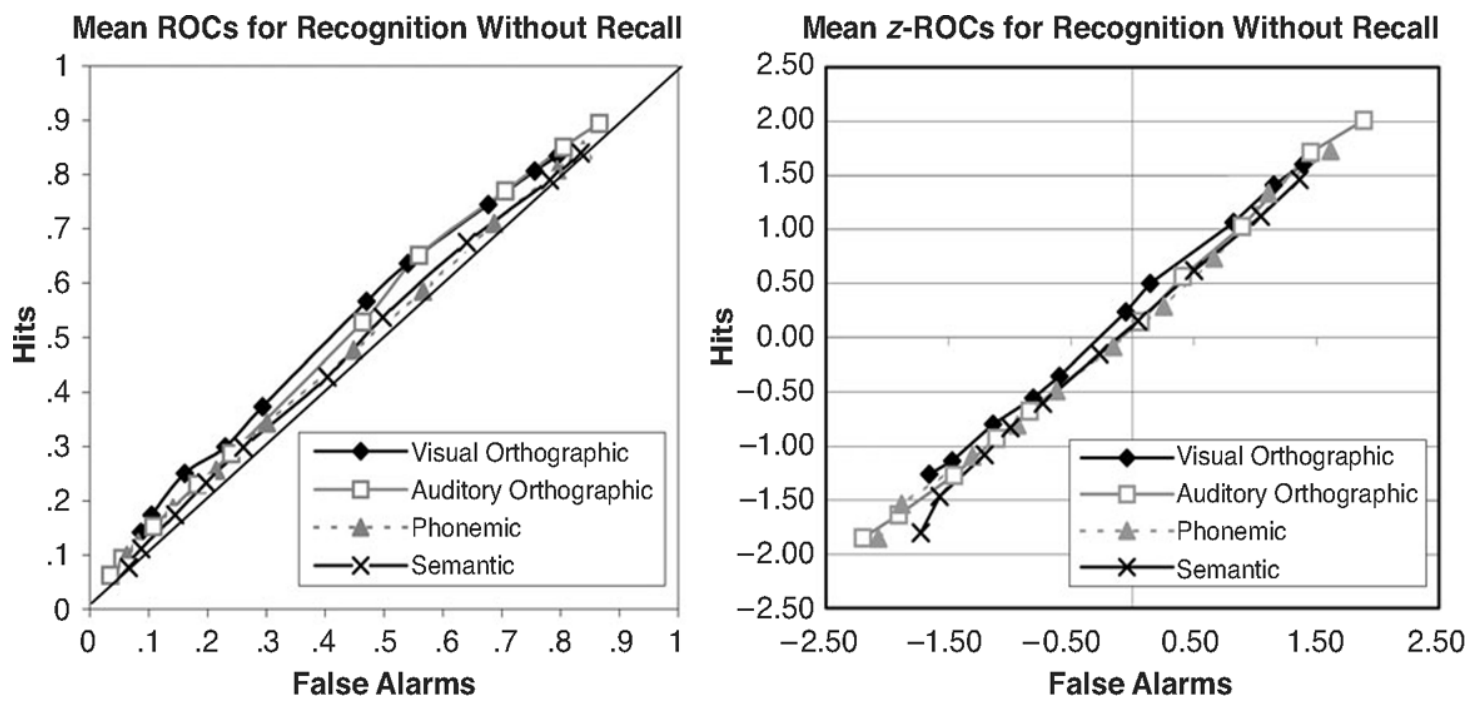

Figure 1. In the left panel are the mean receiver operating characteristics (ROCs) for recognition in the absence of cued recall for all four experimental conditions. In the right panel are the mean $z$-ROCs. 
As is consistent with the Gaussian equal-variance signal detection model of familiarity, the $z$-ROC slopes were very near 1.0 in all of the experimental conditions reported here. It is worth noting that the intercept values (commonly used as estimates of discrimination) are ordered across experimental conditions in the same way as the difference values (the difference between the mean rating for cues of studied items and that for cues of nonstudied items in each condition) that are shown in Table 2 (orthographic cue $>$ modality switch/orthographic cue $>$ rhyme cue $>$ semantic cue).

Some might be concerned that the slopes were so close to 1.0 only because, for many participants, discriminability was at or below zero. When discriminability is at zero, the slope of the $z$-ROC may be forced to equal 1.0 because the signal and noise distributions overlap completely. To address this concern, some researchers have used intercepts of .10 as the cutoff point; those participants whose intercepts fall below .10 are excluded from analysis (e.g., Hirshman \& Hostetter, 2000). Therefore, to address this concern in the present study, the $z$-ROC data for just those participants whose intercepts were greater than .10 were also examined separately. When only these participants were included in the analysis, slope and intercept values were .98 and $.43, .97$ and $.31, .95$ and .30 , and .99 and .31 , respectively. ${ }^{3}$

\section{DISCUSSION}

In the present study, it is shown that participants can base episodic recognition judgments on only an isolated set of a word's features, even when they cannot access the whole-word representation from memory. When participants could not identify the targets corresponding to cues given at test, they gave higher resemblance ratings to cues whose targets had appeared at study than to cues whose targets had not appeared at study. This recognition without recall occurred when the cues resembled studied items orthographically (first and second variations), phonologically (third variation), and semantically (fourth variation).

This ability to recognize in the absence of cued recall may reflect the use of a familiarity process in recognition. Familiarity-based recognition is often described in terms of signal detection theory, according to which the recognition that a particular item or event was experienced recently arises from an assessment of the familiarity signal that it evokes. Exposure to items at study is thought to lead to a temporary increase in their level of familiarity in memory. Thus, the items on the subsequent

Table 3

$z$-ROC $R^{2}$ Values, Slopes, and Intercepts for Recognition Without Cued Recall

\begin{tabular}{llcc}
\hline \multicolumn{1}{c}{ Condition } & $R^{2}$ & Slope & Intercept \\
\hline Orthographic cue & .998 & .96 & .28 \\
Modality switch/orthographic cue & .998 & .97 & .18 \\
Rhyme cue & .996 & .96 & .14 \\
Semantic cue & .997 & 1.01 & .10 \\
\hline
\end{tabular}

test list that were studied will tend to evoke a greater sense of familiarity than will the items that were not studied. If the familiarity signal evoked by a particular test item is above some set criterion, a positive recognition judgment will be given, and if it is below that criterion, a negative recognition judgment will be given.

The present study examined how well the simplest form of signal detection theory, known as the Gaussian equal-variance model, can describe recognition without recall. This model predicts that the $z$-ROCs for recognition should be linear with a slope of 1.0. As is consistent with the idea that recognition without recall reflects the use of an equal-variance signal detection process, in all of the experimental conditions reported here, the $z$-ROCs for recognition without cued recall were linear, with a slope of near 1.0 (as shown in Table 3).

Many theories of familiarity include a role of separable item features in the process. For example, according to the global matching approach (see Clark \& Gronlund, 1996, for review), a test item's familiarity is determined by the degree of match between its features and those from studied episodes that have been stored in memory. However, few studies have addressed the question of what these features might be, particularly in single-word recognition. The present results suggest that the orthographic, phonological, and semantic characteristics of words are features that can give rise to feelings of familiarity.

Furthermore, the present technique for examining recognition without recall may prove useful as a means of separating familiarity from recollection in studies of recognition. Although a number of techniques currently exist for separating these two processes (see Yonelinas, 2002 , for a review), none of the currently available techniques allow for a direct examination of the role of specific types of item features in feelings of familiarity. The present technique, therefore, may be especially useful for this purpose.

This technique may also be useful for examining the nature of the relationship between familiarity and retrieval. The present findings are consistent with the idea that familiarity and recollection are independent, but they do not directly address the question of exactly how familiarity and retrieval relate to one another. The familiarity distribution for studied items, after recalled items are removed from the pool, has the same variability as does the familiarity distribution for nonstudied items. If familiarity and recall were not independent, one might expect the variability of the studied distribution to be less than that of the nonstudied distribution (because the items highest in familiarity might be recalled more often and thus removed from the pool of studied items under consideration). Still, familiarity may be a step that occurs along the way to recalling an item. For example, people may spend more time attempting to generate answers for cues resembling studied items than for cues resembling nonstudied items, due to their familiarity. Such a relationship could be examined in future research using this task. 


\section{REFERENCES}

Blaxton, T. A. (1989). Investigating dissociations among memory measures: Support for a transfer-appropriate processing framework. Journal of Experimental Psychology: Learning, Memory, \& Cognition, 15, 657-668.

CLARK, S. E., \& GRONLUND, S. D. (1996). Global matching models of recognition memory: How the models match the data. Psychonomic Bulletin \& Review, 3, 37-60.

HintzMan, D. L., \& CuRRan, T. (1994). Retrieval dynamics of recognition and frequency judgments: Evidence for separate processes of familiarity and recall. Journal of Memory \& Language, 33, 1-18.

Hirshman, E., \& Hostetter, M. (2000). Using ROC curves to test models of recognition memory: The relationship between presentation duration and slope. Memory \& Cognition, 28, 161-166.

JoNES, T. C., \& JACOBY, L. L. (2001). Feature and conjunction errors in recognition memory: Evidence for dual-process theory. Journal of Memory \& Language, 45, 82-102.

PEYNIRCIOǦLU, Z. F. (1990). A feeling-of-recognition without identification. Journal of Memory \& Language, 29, 493-500.

RatCliff, R., McKoON, G., \& Tindall, M. (1994). Empirical generality of data from recognition memory receiver-operating characteristic functions and implications for global memory models. Journal of Experimental Psychology: Learning, Memory, \& Cognition, 20, 763-785.

YoNELINAS, A. P. (1994). Receiver-operating characteristics in recognition memory: Evidence for a dual-process model. Journal of Experimental Psychology: Learning, Memory, \& Cognition, 20, 1341-1354.

YonELINAS, A. P. (2002). The nature of recollection and familiarity: A review of 30 years of research. Journal of Memory \& Language, $\mathbf{4 6}$, 441-517.

\section{NOTES}

1. The present study only sought to determine the types of word features that, when presented in isolation, could give rise to feelings of familiarity; no attempt was made to tease apart the various contributions of specific types of word features to overall feelings of familiarity in normal word recognition.

2. Because four study-test segments were used in the present study, the data were analyzed for possible practice effects. With the exception of the auditory orthographic variation, in which discrimination appears to have improved from one study-test segment to the next $[F(1,20)=$ $3.50, p=.076]$, none were found. In the auditory orthographic variation, as participants became more experienced with the orthographic cued recall task, they may have followed a strategy of visualizing the orthography of the words as they were hearing them at study, which may have helped them in later recognizing the orthographic similarity of a cue to a studied word in the absence of recall.

3. Further support for the claim that the present slopes are not the result of low discrimination came from correlations computed between slopes and intercepts across participants. The only significant correlation found was in the visual orthographic condition $[r(18)=.479, p<$ $.05]$, and this correlation was positive, rather than negative; that is, as discrimination decreased, so did the slope. There were no significant correlations found for the auditory orthographic condition $[r(20)=$ $-.031, \mathrm{n} . \mathrm{s}$.], for the phonemic condition $[r(33)=-.040$, n.s.], or for the semantic condition $[r(28)=.168$, n.s. $]$.

(Manuscript received February 21, 2003; revision accepted for publication July 7,2003 .) 